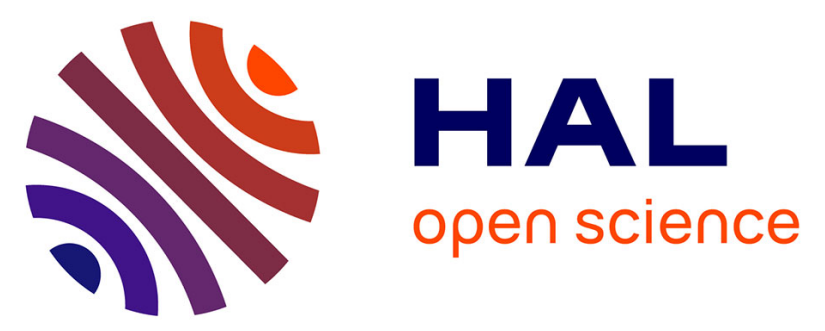

\title{
Characterization of the porous structure of a non-woven fibrous medium for air filtration at local and global scales using porosimetry and X-ray micro-tomography
}

Félicie Theron, Elisabeth Lys, Aurélie Joubert, François Bertrand, Laurence Le Coq

\section{To cite this version:}

Félicie Theron, Elisabeth Lys, Aurélie Joubert, François Bertrand, Laurence Le Coq. Characterization of the porous structure of a non-woven fibrous medium for air filtration at local and global scales using porosimetry and X-ray micro-tomography. Powder Technology, 2017, 320, pp.295-303. 10.1016/j.powtec.2017.07.020 . hal-01700199

\section{HAL Id: hal-01700199 \\ https://hal.science/hal-01700199}

Submitted on 5 Jul 2018

HAL is a multi-disciplinary open access archive for the deposit and dissemination of scientific research documents, whether they are published or not. The documents may come from teaching and research institutions in France or abroad, or from public or private research centers.
L'archive ouverte pluridisciplinaire HAL, est destinée au dépôt et à la diffusion de documents scientifiques de niveau recherche, publiés ou non, émanant des établissements d'enseignement et de recherche français ou étrangers, des laboratoires publics ou privés. 


\title{
Characterization of the porous structure of a non-woven fibrous medium for air filtration at local and global scales using porosimetry and X-ray micro-tomography
}

\author{
Félicie Théron ${ }^{\mathrm{a}, *}$, Elisabeth Lys ${ }^{\mathrm{b}}$, Aurélie Joubert ${ }^{\mathrm{a}}$, François Bertrand ${ }^{\mathrm{c}}$, Laurence Le Coq ${ }^{\mathrm{a}}$ \\ a GEPEA, UMR CNRS 6144, IMT Atlantique, CS 20722, 44307 Nantes, France \\ b SUBATECH, UMR CNRS 6457, IMT Atlantique, CS 20722, 44307 Nantes, France \\ c GeM, UMR CNRS 6183, Ecole Centrale de Nantes, University of Nantes, 1 rue de la Noe, 44321 Nantes, France
}

Keywords:

Fibrous medium

Porous structure

Mercury porosimetry

X-ray micro-tomography

\begin{abstract}
A B S T R A C T
The present study focuses on complementary experimental methods to characterize the structural properties of a high performance composite medium made of a polymer membrane laminated to a fibrous support, and used for air filtration, at global and local scales. This two-scale approach aims to evaluate the in-plane homogeneity of the properties of the two layers of the medium.

The structure of the medium is observed in 2D by Scanning Electron Microscopy, and in 3D by X-ray microtomography. Those observations enable for example the isotropic/anisotropic nature of each layer of the medium to be evaluated. Some characteristics, like the fibrous support fiber diameters and the thickness of each layer, are also quantified.

The porous structures of each layer are first described from a global standpoint in terms of mean porosity and pore size distribution obtained by the mercury porosimetry technique. Micro-tomography data are treated to calculate mean porosity values of each layer that are compared to those obtained by mercury porosimetry, and to represent porosity profiles through each layer thickness to give orders of magnitude of the through-plane porosity gradients that they exhibit. In order to evaluate the in-plane homogeneity of the fibrous support porous structure, micro-tomography data are then used to estimate local porosity values from analyses carried out on two different sample areas. The pore sizes of the polymer membrane are evaluated by analysis of 2D images obtained by micro-tomography, and a good agreement is highlighted with those obtained by mercury porosimetry.
\end{abstract}

\section{Introduction}

Filtration with fibrous filters is the most common technology used in in-duct ventilation systems. The significant number of processes and fiber materials, including polymer types, used to manufacture them leads to wide ranges in thickness, fiber morphology, size distribution, diameter to length ratio, fiber orientation, pore size distribution, mean porosity, through-plane porosity distribution... In the case of significant local variations in these properties, the media can exhibit a certain level of anisotropy and heterogeneity. It is thus of major importance to be able to characterize the structural properties of fibrous media as they directly influence their filtration performances.

\footnotetext{
* Corresponding author.

E-mail addresses: felicie.theron@imt-atlantique.fr (F. Théron), elisabeth.lys@subatech.in2p3.fr (E. Lys), aurelie.joubert@imt-atlantique.fr (A. Joubert), francois.bertrand@ec-nantes.fr (F. Bertrand), laurence.le-coq@imt-atlantique.fr (L. Le Coq).
}

The challenge of fibrous filter design is to find a compromise between a high collection efficiency for a given particle size range and a low pressure drop, which is directly related to the permeability of the medium. The models that predict the permeability of fibrous media generally require mean porosity and fiber diameter. Some studies have investigated the influence of some properties separately by numerical methodologies based on virtual media [1,2]. However, the rather anisotropic, heterogeneous and soft nature of real fibrous media can lead to deviations between measured and predicted permeability.

The characterization of both the solid and porous structures of fibrous media is thus an important issue to evaluate the variations exhibited by their properties. For this purpose, relevant experimental methods have to be selected by taking into consideration the characteristic length scales of the parameters investigated. Moreover, the characterization of quantitative parameters must be carried out on relevant sample sizes in order to be representative of the average properties of the media.

Only a few studies have dealt with the porous structure characterization of non-woven media. Le Coq [3] worked on the 
characterization of the structural parameters of heterogeneous glass fiber non-wovens by mercury porosimetry and image analysis. A good agreement was found for the mean porosity obtained by both methods. Manickam \& McCutcheon [4] compared the pore size distributions of polyester non-wovens and electrospun polyacrylonitrile nanofibers obtained by mercury porosimetry and capillary flow porometry. They also determined the average porosity by Xray computed tomography.

In the last few years, some studies dealing with the use of X-ray micro-tomography to characterize fibrous materials have been reported in the literature. Different data about the porous or fibrous structure are derived from such measurements. Koivu et al. [5] and Soltani et al. [6] developed X-ray micro-computed tomography-based CFD simulation methodologies to determine the three components of the permeability of a felt material made of plastic fibers and of a hand sheet paper made of chemical hardwood pulp, and the in-plane permeability of a nonwoven polypropylene medium respectively. Gervais et al. [7] used this technique to create representative computational domains to determine the permeability and simulate the clogging of a glass fiber media. Charvet et al. [8] performed X-ray holotomography to observe the three-dimensional structure of a fibrous filter containing cellulose fibers and to determine the liquid distribution in the filter clogged with a liquid aerosol of di-ethyl-hexyl-sebacate (DEHS). Jackiewicz et al. [9] carried out X-ray micro-tomography analyses to observe the in-depth structure of silica particle deposits in two melt-blown polypropylene materials and calculate their porosity profiles. Thibault and Bloch [10] used micro-tomography to observe and characterize the 3D structure of a nonwoven papermaker felt through the calculation of the mean porosity, and the representation of porosity profiles. Rolland du Roscoat et al. [11] studied the porous structure of four industrial paper materials in terms of mean porosity and porosity profile. Ramaswamy et al. [12] visualized and described the 3D structure of polymeric fabrics used in paper manufacture through the calculation of specific area, tortuosity and yarn radii distribution in the two principal orthogonal directions. Huang et al. [13] investigated the solid and void phase morphology of porous metal fiber sintered sheet using $\mathrm{X}$-ray tomography. They reported statistical data in terms of fiber orientation, length and tortuosity, as well as porosity, tortuosity and pore diameters. They also carried out Latice Boltzmann simulations in order to determine the medium transverse permeability. Thus, except for Huang et al. [13], no authors have used micro-tomography data to determine the pore size of fibrous media, and no comparison has been made between the pore sizes obtained by micro-tomography and mercury porosimetry.

The aim of the present study is to demonstrate the complementarity between several experimental methods to describe the solid and porous structures of an original two-layer medium. In order to address the inplane heterogeneity of its structural properties, special attention is paid to each layer to characterize them from both a global and a local standpoint, and to compare the results obtained at each scale. The different methods used to perform the different characterizations are presented in Section 2. The solid structures of each layer are observed in 2D and 3D by SEM and X-ray micro-tomography, respectively, at relevant resolutions, in Section 3.1. 3D reconstructions of microtomography data also enable their respective thicknesses to be estimated. The value obtained for the fibrous support is compared to that obtained by a facility developed for low-pressure measurements on paper samples.
In Section 3.2, the porous structures of each layer are first described from a global standpoint in terms of mean porosity and pore size distribution obtained by the mercury porosimetry technique. Microtomography data are then treated to calculate mean porosity values of each layer, and to give orders of magnitude of the through-plane porosity gradients that they exhibit thanks to the representation of porosity profiles through each layer thickness. In order to evaluate the in-plane homogeneity of the fibrous support porous structure, micro-tomography data are used to estimate local porosity values from analyses carried out on different sample areas. The pore sizes of the polymer membrane are also evaluated by analysis of some 2D images obtained by microtomography, and comparison to those obtained by mercury porosimetry.

\section{Materials and methods}

\subsection{The studied medium}

The two-layer medium studied is called Arioso ${ }^{\circledR}$ and is commercialized by the Lydall Company. In order to reach performances of an E10 type (according to the European standard EN1822) while lowering the pressure drop, it consists of a fibrous support made of polyester fibers, on which a polyethylene membrane is laminated. Samples of the complete medium, as well as of each layer separately were supplied by the manufacturer to carry out the different characterizations.

\subsection{Characterization methods}

\subsubsection{Scanning Electron Microscopy}

Each layer of the medium was observed by Scanning Electron Microscopy using a JEOL 5800 apparatus with $5 \mathrm{~nm}$ resolution. The fiber diameter of the fibrous support was determined using the Image J software.

\subsubsection{Mercury porosimetry}

Mercury porosimetry measurements were carried out using the Autoport IV Micromeritics apparatus, for samples of both layers of the medium separately. The order of magnitude of the sample areas was $100 \mathrm{~mm}^{2}$. The mercury volume penetrating the porous medium was measured at different pressures in order to obtain a porosity versus pore size curve according to the Washburn relationship:

$\mathrm{D}_{\mathrm{p}}=\frac{-4 \cdot \gamma \cdot \cos \theta}{\mathrm{P}_{\mathrm{c}}}$

where $D_{p}, \gamma, \theta$ and $P_{c}$ are the pore diameter, the mercury superficial tension, the solid/liquid contact angle and the pressure, respectively. For the fibrous support samples the analyses involved 167 pressure points ranging from 1086 to $2.068 \times 10^{8} \mathrm{~Pa}$, which corresponds to a pore size range of $8 \mathrm{~nm}-1593 \mu \mathrm{m}$. For the membrane samples the analyses involved 204 points ranging from 1068 to $2.068 \times 10^{8} \mathrm{~Pa}$, which corresponds to a pore size range of $8 \mathrm{~nm}-1619 \mu \mathrm{m}$. For all the analyses the equilibration time at each pressure step was fixed to $10 \mathrm{~s}$. The surface tension and contact angle used for the polyester fibers (fibrous support) and the polyethylene membrane were $0.485 \mathrm{~N} \cdot \mathrm{m}^{-1}$ and $153^{\circ}$, respectively.

Table 1

Experimental conditions of X-ray micro-tomography analyses.

\begin{tabular}{|c|c|c|c|c|c|c|}
\hline Sample reference & Layers involved & Observation facility & Resolution ( $\mu \mathrm{m} /$ pixel $)$ & Sample diameter (mm) & Analyzed zone diameter (mm) & Analyzed zone area $\left(\mathrm{mm}^{2}\right)$ \\
\hline $\mathrm{CM}$ & Complete medium & ID19, ESRF & 0.16 & 4 & 0.41 & 0.13 \\
\hline FSa & Fibrous support & Xradia Micro XCT-400 & 1.99 & 3 & 3 & 7 \\
\hline FSb & & & 9.59 & 10 & 10 & 79 \\
\hline
\end{tabular}




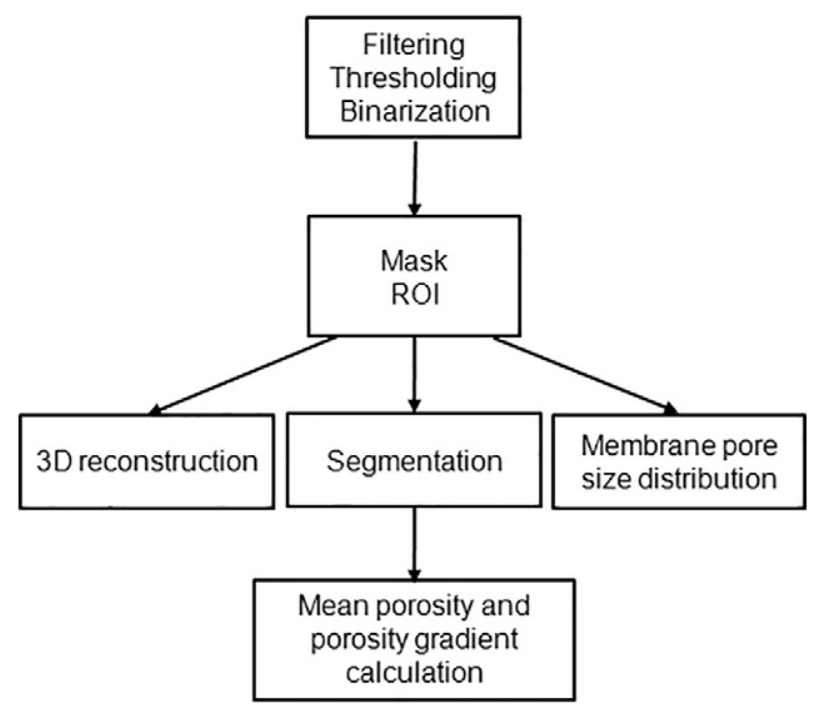

Fig. 1. Processes applied to the $2 \mathrm{D}$ images obtained by X-ray micro-tomography.

\subsubsection{X-ray micro-tomography}

Three different X-ray micro-tomography analyses were carried out on circular samples, either on the complete medium or on the fibrous support, at different spatial resolutions consistent with the respective characteristic length scales of the solid structure of both layers. Two different analyses were performed on the fibrous support at two different resolutions, and thus on two different sample diameters, in order to evaluate the influence of the observed sample area on the results obtained. The main experimental conditions of these analyses are given in Table 1.

The analysis of the complete medium (on the sample named $\mathrm{CM}$ ) was performed on the ID19 beamline of the European Synchrotron Radiation Facility (ESRF, Grenoble, France) with a high spatial resolution of $0.16 \mu \mathrm{m} /$ pixel, relevant to the smallest characteristic length scale of the complete medium, i.e. exhibited by the membrane. The beamline energy was $19 \mathrm{keV}$. A $2560 \times 2560$ pixel $^{2}$ camera equipped with a $\times 40$ zoom lens was used in order to reach a pixel size of 0.16 $\mu \mathrm{m}$. The complete medium observation involved 3202 tomograms.

The two analyses carried out on samples of the fibrous support were performed with an Xradia Micro XCT-400 tomograph at 1.99 and 9.59 $\mu \mathrm{m} /$ pixel resolutions, respectively, on samples named FSa and FSb. The beamline energy was $40 \mathrm{kV}$. A $2048 \times 2048$ pixel $^{2}$ camera was used, and equipped with $\mathrm{a} \times 4$ zoom lens for the analysis performed at the smallest resolution. These observations involved 440 and 97 tomograms, respectively.

In the Table 1 the analyzed zone diameter corresponds to the higher achievable value according to the chosen resolution. For the two analyses carried out on the fibrous support the analyzed zone diameters correspond to the sample diameters respectively. Due the high resolution chosen for the measurement performed on the complete medium, the higher achievable analyzed zone diameter is very small $(410 \mu \mathrm{m})$. That is why the observation was realized only on a small zone of a sample of higher diameter ( $4 \mathrm{~mm}$ ).

The $2 \mathrm{D}$ images obtained by these analyses were reconstructed and processed using different software depending on the targeted information (cf. Fig. 1); these were chosen in order to reduce calculation times as much as possible. The Mevislab software enabled 3D reconstructions of samples to be built from stacking 2D raw images, and the pore size of the membrane layer to be calculated for the analysis carried out on the sample CM (cf. Section 3.2.2). In order to determine the mean porosity and through-plane porosity gradient of both layers, circular Regions Of Interest were defined and applied to the 2D raw images using the Matlab software. In parallel to this geometrical processing, the FIJI software was used to carry out the pre-processing of these images. After having adjusted the brightness and contrast of the pictures, the first step consisted in frequency filtering by using a pass band [14] and a median filter that allow the residual noise to be eliminated. Then the thresholding step enabled to convert greyscale into binarized pictures in order to distinguish void and solid fractions. For that purpose the Triangle method due to Zack et al. [15] was used. The threshold value was adapted for each sample. Then the Mevislab software was used to segment the 2D images, which enabled the pixels corresponding to fibers or void to be quantified for each picture.

Fig. 2 compares the area of the samples used to perform the different characterizations from which the porosity values of each layer were quantified, i.e. mercury porosimetry and X-ray micro-tomography. The sample areas involved in mercury porosimetry measurements were of the same order of magnitude as the sample FSb, which enabled a proper comparison between the mean porosity of the fibrous support obtained by both techniques.

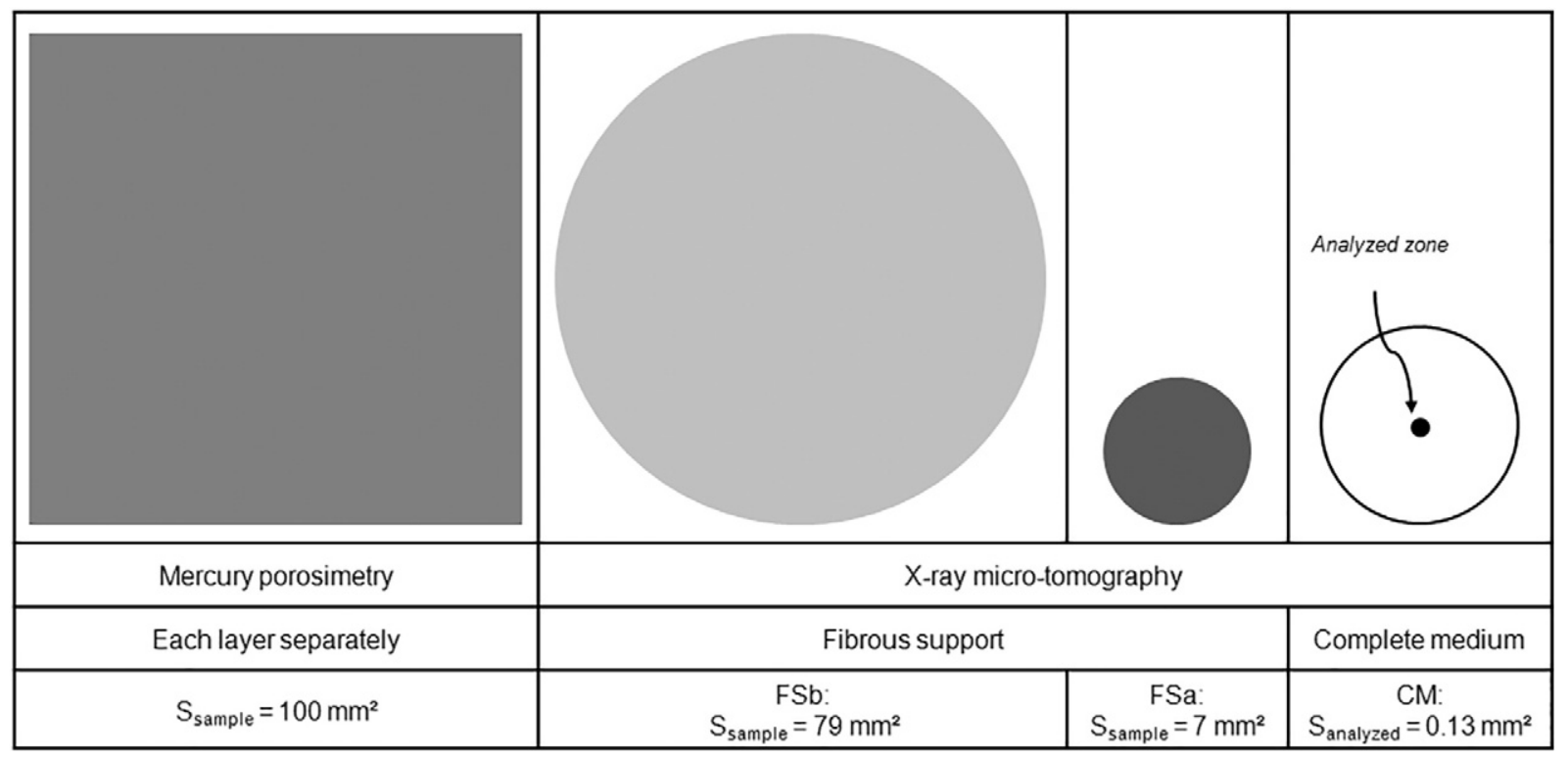

Fig. 2. Comparison of the sample areas involved in the different characterizations used to quantify the porosity values of each layer. 

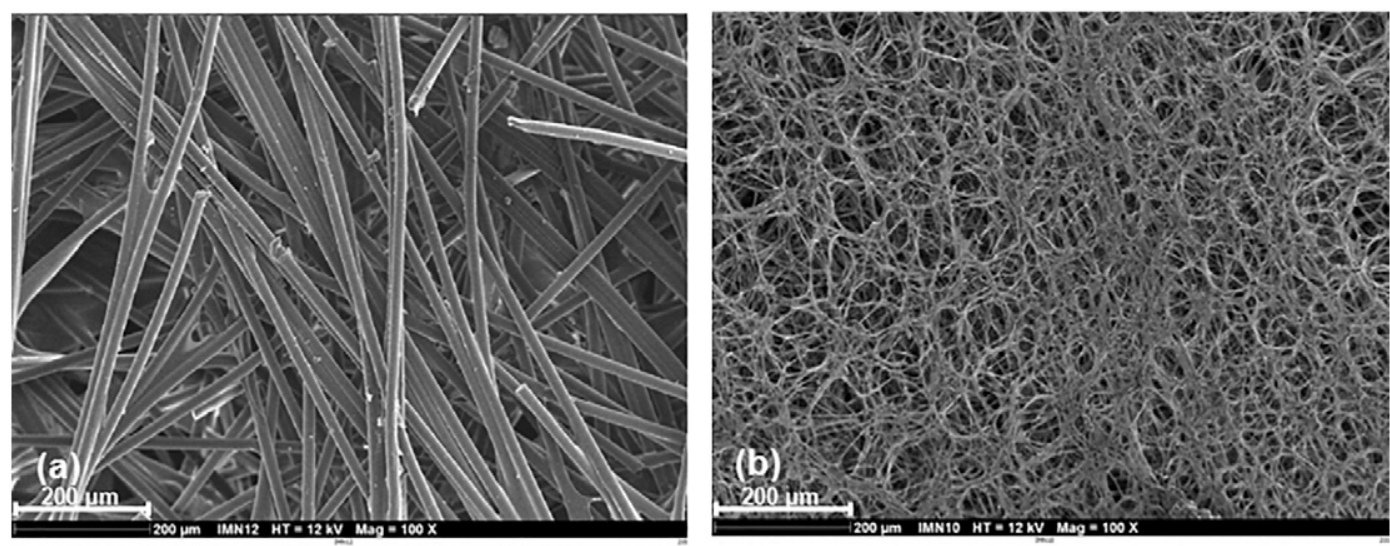

Fig. 3. Scanning Electron Microscopy images of each layer of the medium at $\times 100$ magnification: (a) fibrous support, (b) polyethylene membrane.

\subsubsection{Thickness measurements}

The thicknesses of the complete medium and the fibrous support were measured by the facility developed at the LGP2 Laboratory and used by Zerrouati et al. [16] to measure the thickness of paper samples at low pressures. These measurements were carried out on eight 10 $\times 10 \mathrm{~cm}^{2}$ randomly distributed zones over a total surface area of $1250 \mathrm{~cm}^{2}$. The pressure was fixed at the lowest achievable value of $2.1 \mathrm{mbar}$, in order to make a relevant comparison with the thickness estimated for the fibrous support from micro-tomography analyses, which involved relaxed samples.

\section{Results and discussion}

\subsection{Characterization of the solid structure}

\subsubsection{SEM observations}

Fig. 3 shows the observations of each layer of the medium at the same magnification. They reveal the distinct solid structures of each layer, as well as their characteristic length scales. The fibrous support is made of cylindrical fibers arranged in parallel planes, which thus lead to 3D anisotropy. The fibers exhibit rather homogeneous diameters, which were estimated by image processing. From the measurements carried out on 212 fibers, a value of $24.0 \pm 0.1 \mu \mathrm{m}$ was obtained.

The polymer membrane consists of a solid array that can be observed at higher magnification in Fig. 4. From this figure, it appears that the width of filaments is of the order of magnitude of $5 \mu \mathrm{m}$.

\subsection{2. $3 D$ reconstruction of $X$-ray micro-tomography images}

In addition to the 2D images of each layer obtained by SEM, X-ray micro-tomography analyses provided 3D observations of their respective structures. Fig. 5 shows a 3D reconstruction of the complete medium. From the number of images involved in this reconstruction, it is possible to estimate the mean thickness of the membrane layer, which is around $50 \mu \mathrm{m}$ locally. This value is a local one as it was obtained for an analyzed zone of the CM sample of $410 \mu \mathrm{m}$ diameter. This thickness estimation was not done for the complete medium as the ratio of the analyzed zone diameter to the fibrous support mean fiber diameter is only 17 , which can lead to an uncertainty in fibrous media surface area detection.

Like the SEM observations, the 3D view shows the significant difference between both layers (membrane and fibrous support) in terms of the characteristic length scales of the porous structures. For the observed zone diameter of $410 \mu \mathrm{m}$, the interface between the fibrous support and the membrane only concerns a few fibers.

Fig. 6 presents the 3D reconstructions of the fibrous support obtained from the two micro-tomography analyses carried out at two different resolutions, and thus on two distinct sample diameters. Like the SEM observations, they show that the fibers are homogeneous in diameter, mainly oriented in the medium plane direction, and stacked on top of each other, which confirms its rather anisotropic nature. The mean thicknesses of samples FSb and FSa are $921 \mu \mathrm{m}$ with a $10 \mu \mathrm{m}$ precision and $878 \mu \mathrm{m}$ with a $2 \mu \mathrm{m}$ precision, respectively. This represents a 5\% thickness deviation for an 11.2 ratio between both sample areas. The sample FSa, of smaller diameter ( $3 \mathrm{~mm}$ ), can thus be considered representative of the fibrous support in terms of thickness.

\subsubsection{Thickness measurements}

The thickness measured for the fibrous support was $918 \pm 22 \mu \mathrm{m}$. The values estimated by the two micro-tomography analyses are thus in good agreement with this result.

For the complete medium, a thickness of $832 \pm 9 \mu \mathrm{m}$ was measured, which is less than the fibrous support thickness. This may be due to deviation in the fibrous support manufacturing process, as the analysis carried out on the fibrous support did not involve the same batch as that of the complete medium sample.

\subsection{Characterization of the porous structure}

\subsubsection{Mercury porosimetry}

3.2.1.1. Fibrous support. The mercury porosimetry results obtained for the two analyses carried out on the fibrous support are presented in Fig. 7 in terms of differential porosity as a function of pore diameter. The distributions exhibit a minimum pore size of around $100 \mu \mathrm{m}$, and

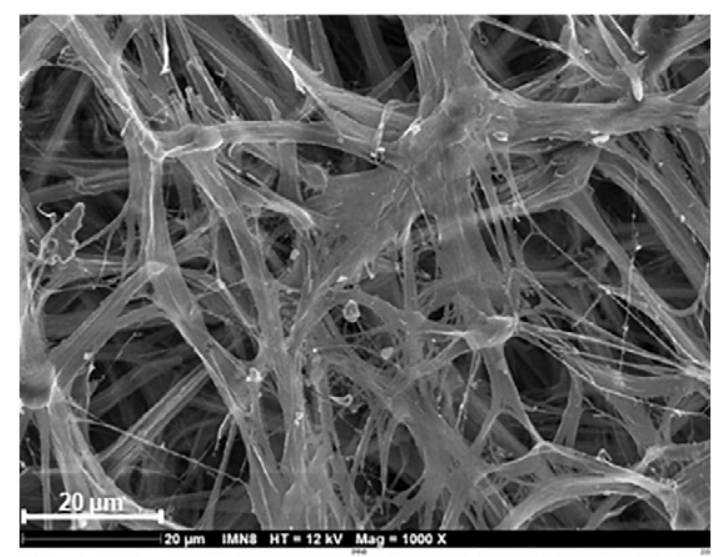

Fig. 4. Scanning Electron Microscopy images of the polyethylene membrane at $\times 1000$ magnification. 

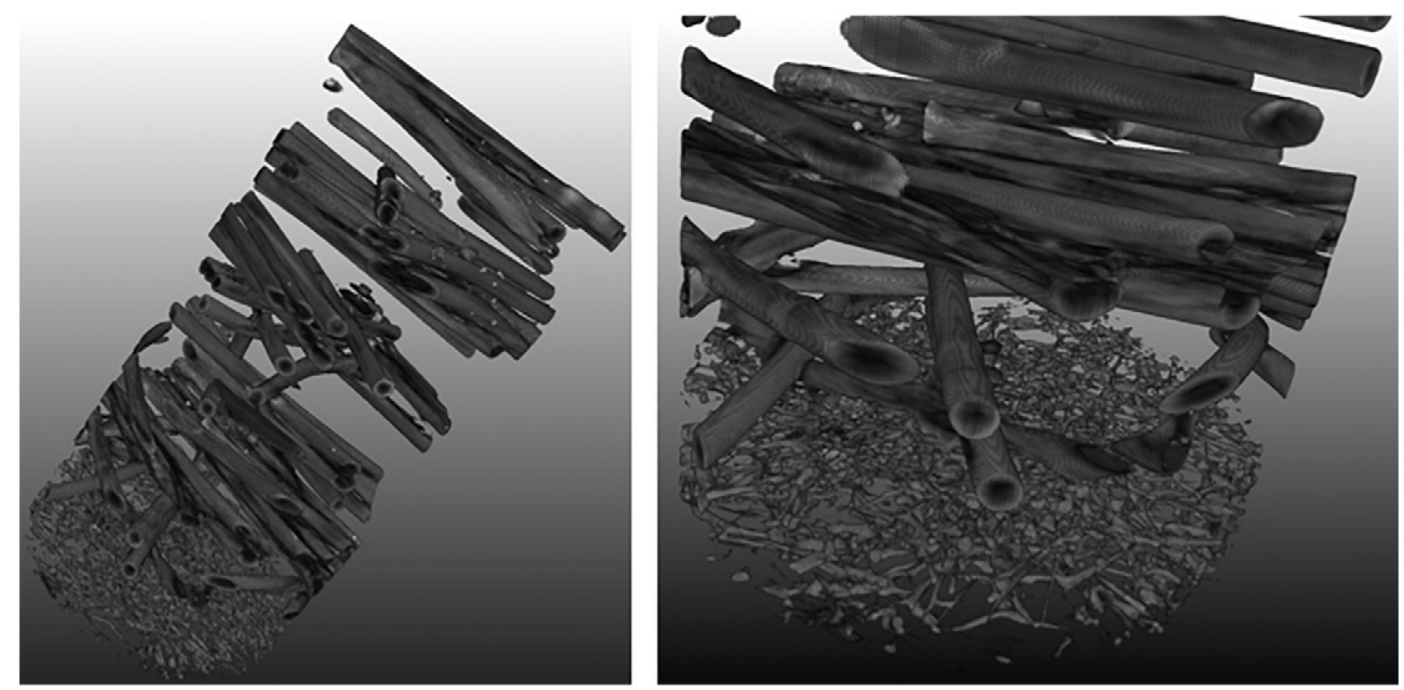

Fig. 5. 3D reconstruction of the complete medium from the micro-tomography analysis carried out on sample CM.
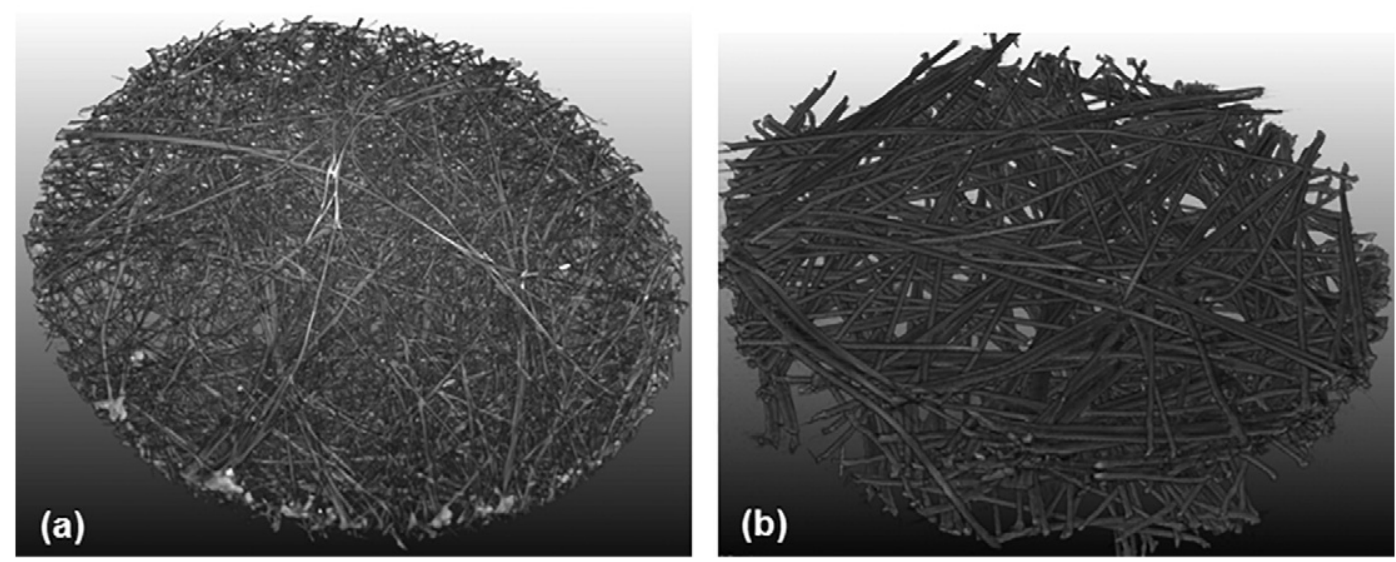

Fig. 6. 3D reconstructions of the fibrous support from micro-tomography analyses carried out on: (a) sample FSb, (b) sample FSa.

two interpenetrated populations characterized by modes of 255 and $860 \mu \mathrm{m}$, respectively. The total porosity obtained is $85 \pm 1 \%$ for the two measurements.

These distributions were modeled using a probability distribution of porosity versus pore size as proposed by Le Coq [3]:

$\varepsilon\left(D_{p, j}\right)=\sum_{i=1}^{k} \frac{\varepsilon_{i}^{\max }}{\left(\left(\frac{D_{p, j}}{D_{p, i, \bmod }}\right)^{\alpha_{i}}+1\right)}$

where $\varepsilon_{\mathrm{i}}^{\max }$ and $\mathrm{D}_{\mathrm{p}, \mathrm{i}, \mathrm{mod}}$ are the maximum porosity and modal pore size of each level (i) of the pore size distribution, respectively. $\alpha_{\mathrm{i}}$ is the slope attenuation coefficient at the inflexion point of the cumulative porosity curve for the pore size distribution $i$. The parameters were obtained by fitting the model to the experimental curve. The porosities of the two levels characterized by modal pore sizes of $255 \mu \mathrm{m}$ and $860 \mu \mathrm{m}$ are $58 \%$ and $27 \%$, respectively.

3.2.1.2. Polymer membrane. The results obtained for the three analyses carried out on the polyethylene membrane are presented in Fig. 8. Some deviations appear between the three measurements at the largest diameters (between 10 and $1000 \mu \mathrm{m}$ ) which can be explained by a measurement artifact ("step" of penetrated mercury volume) between the first and second points for both measures 2 and 3. Such a phenomenon can reveal a rearrangement of the sample inside the measurement chamber when the mercury starts to penetrate the measurement volume. Nevertheless, these three measurements highlight minimum pore diameters of about $1 \mu \mathrm{m}$, a main mode for the smallest diameters centered on $8 \mu \mathrm{m}$, and a second spread population interpenetrated

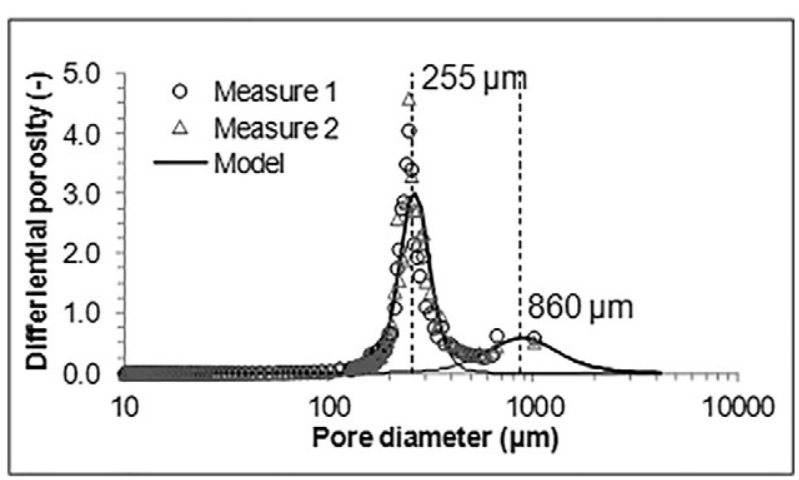

Fig. 7. Pore size distribution of the fibrous support 


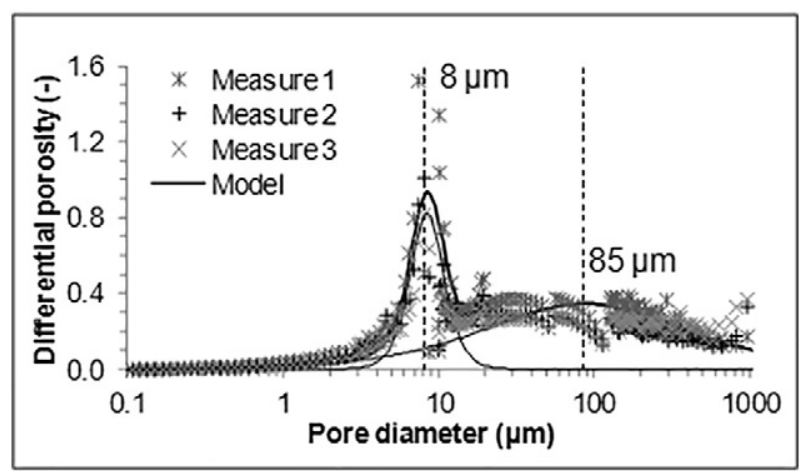

Fig. 8. Pore size distribution of the polymer membrane.

with the first one, which covers pore sizes ranging from around $20 \mu \mathrm{m}$ to the maximum detection limit, which is about $1-2 \mathrm{~mm}$. The total porosity obtained for this polymer membrane ranges between 81 and $87 \%$ for the three measurements.

\subsubsection{X-ray micro-tomography}

Mercury porosimetry enables the average porous structure of the samples to be depicted. In this section, these results are compared and complemented by the mean and local information achievable by X-ray micro-tomography.

3.2.2.1. Fibrous support. By processing the $2 \mathrm{D}$ images obtained by microtomography, the evolution of the porosity through the fibrous support thickness, i.e. the porosity profile across the medium, could be represented. For this purpose, the porosity was calculated for each image, in order to represent the through-plane porosity profile with a thickness step size equal to the resolution, i.e. $9.59 \mu \mathrm{m}$ and $1.99 \mu \mathrm{m}$ for samples FSb and FSa, respectively. The through-plane porosity profiles obtained are presented in Fig. 9 (a) and (b).

In these two graphs, the grey strip represents the mean porosity range obtained by mercury porosimetry. For each micro-tomography analysis, two mean porosity values were determined: one taking into consideration the complete stack of images containing fibers represented by a solid line, and the second represented by a dotted line for which the last images of each side partly containing fibers were not taken into consideration, in order to evaluate a potential influence of the effect of the medium top and bottom surfaces.

Fig. 9 shows, as expected, that the porosity increases on both sample sides. Fig. 9 (a), corresponding to the largest sample FSb, shows that whether or not the last images containing fibers on each side are taken into consideration, the mean porosity is in good agreement with the porosity range of $84-86 \%$ obtained by mercury porosimetry. In fact, the mean porosity obtained for image stack thicknesses of 921 $\mu \mathrm{m}$ (complete image stack containing fibers) and $680 \mu \mathrm{m}$ (74\% of the complete image stack thickness) is $85.5 \%$ and $83.4 \%$, respectively. Moreover, except on its surface, the porosity fluctuates from 80.9 to $85.7 \%$ around the mean value.

Fig. 9 (b), corresponding to a sample area 11 times smaller than in Fig. 9 (a) (sample FSa), shows higher porosity levels, even inside the medium. In fact, the mean porosity obtained for image stack thicknesses of $878 \mu \mathrm{m}$ (complete image stacks containing fibers) and $570 \mu \mathrm{m}(65 \%$ of the complete image stack thickness) are $91.8 \%$ and $89.8 \%$, respectively. It is also interesting to notice that this profile exhibits a high porosity peak inside the medium, which reaches $94.4 \%$ and corresponds to a thickness of approximately $170 \mu \mathrm{m}$. Except for this peak, the porosity inside the medium fluctuates around $88 \%$ (and exhibits a low porosity peak of $84 \%$ ), which is rather close to the value obtained by mercury porosimetry, and by micro-tomography on a larger sample.

The good agreement between the mean porosity obtained for the sample FSb and that obtained by mercury porosimetry is consistent with similar sample surface areas (cf. Fig. 2).The significant discrepancy in terms of mean porosity obtained from two different sample areas indicates that the sample FSa does not seem representative of the medium average porous structure. This observation may highlight the rather inplane heterogeneous nature of the porous structure of the medium.

For this reason, porosity calculations were also carried out on five non-interpenetrated small circular zones of the sample FSb, taking into account the complete stack of images containing fibers. These zones and the corresponding local porosity values, which enable inplane variations in the porosity to be evaluated, are shown in Fig. 10. These zones are $1.49 \mathrm{~mm}$ in diameter, which corresponds to an area of $1.74 \mathrm{~mm}^{2}$, i.e. 45 times smaller than the total sample area. Such a diameter is thus consistent with the medium mean fiber diameter as it represents 62 times this value $(24 \mu \mathrm{m})$. The average porosity of the five zones is $85.6 \%$, which is in good agreement with the value of $85.5 \%$ obtained for the complete sample. Separately, these zones exhibit porosities ranging from 81.8 to $89.1 \%$, which also indicates that the porous structure of this medium is rather heterogeneous.

3.2.2.2. Polymer membrane. For the polyethylene membrane, the porosity was calculated for each $2 \mathrm{D}$ image obtained for the sample $\mathrm{CM}$. The analyzed zone diameter of $410 \mu \mathrm{m}$ is consistent with the membrane filament size, as it represents 82 times its order of magnitude $(5 \mu \mathrm{m})$. Average values were derived from series of five images, in order to represent the through-plane porosity profile with a thickness step of $0.8 \mu \mathrm{m}$. This profile is represented in Fig. 11 from the fibrous support/membrane interface (zero abscissa) to the membrane surface.

The mean porosity estimated by micro-tomography is 86\%. Fig. 11 shows that the local porosity is rather lower at the support/membrane interface, and then fluctuates around the mean value, from 83 to $89 \%$.
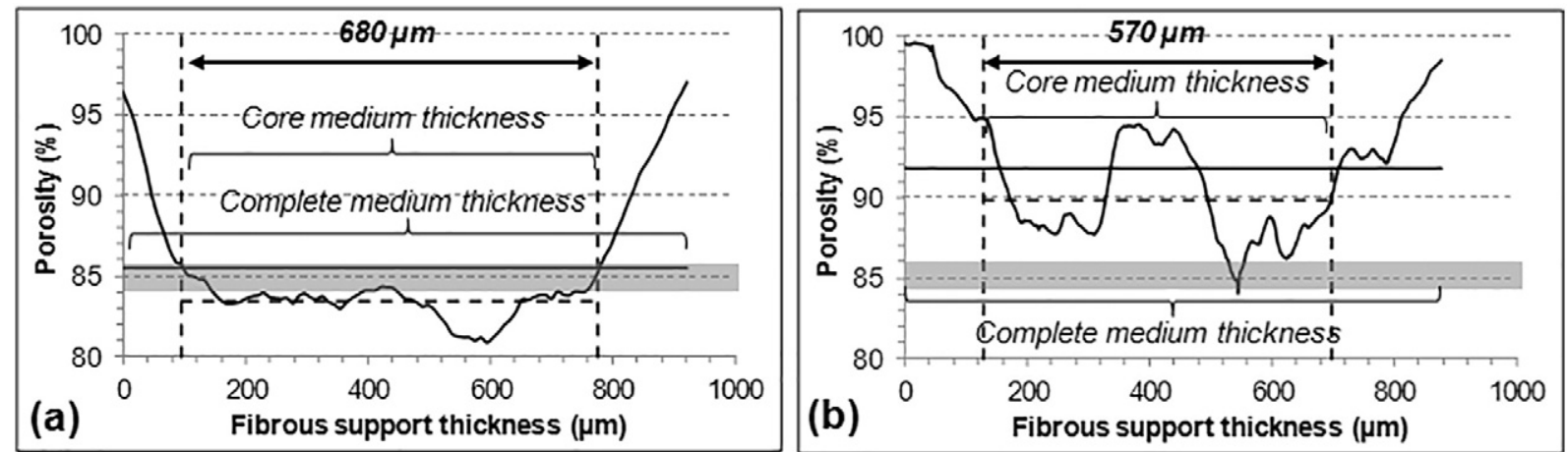

Fig. 9. Through-plane porosity profile for the fibrous support from micro-tomography analyses carried out on: (a) sample FSb, (b) sample FSa. 


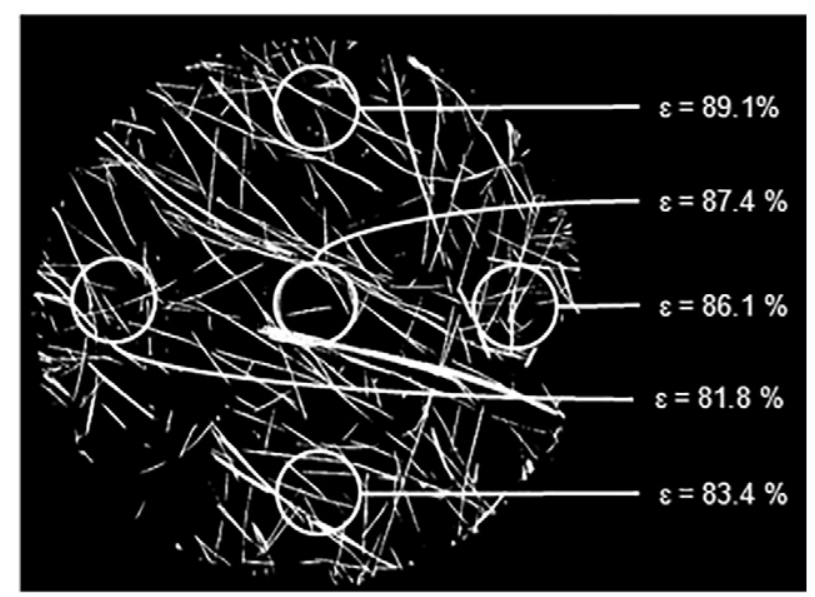

Fig. 10. In-plane variation in the porosity of the $10 \mathrm{~mm}$ diameter sample.

These results are thus in good agreement with the $81-87 \%$ obtained by mercury porosimetry.

Some of the 2D images obtained by micro-tomography corresponding to the polyethylene membrane layer were also processed in order to estimate pore diameters in the medium plane, assuming that the membrane structure is isotropic (cf. Figs. 3 and 5). For that purpose, the pores were cropped on each slice to obtain their area. The diameters of circles of equivalent surface areas were then calculated. An example is presented in Fig. 12.

This methodology was applied to 7 images distributed along the membrane thickness, on a total of 346 cropped pores. Their diameters are comprised between 1.0 and $113.2 \mu \mathrm{m}$.

This methodology was not applied to the fibrous support due to its solid structure. In fact, while the network-like solid structure of the polyethylene membrane enables pores on the in-plane 2D images to be cropped, this is not possible for the fibrous support, which consists of stacked fibers mostly oriented in the medium plane.

Fig. 13 represents the number pore size distribution obtained with this method. These results show that the smallest pore size of around $1 \mu \mathrm{m}$ is in good agreement with that obtained by mercury porosimetry. Most of the counted pores exhibit diameters ranging from several micrometers to several tens of micrometers, which is also consistent with the largest population exhibited by mercury porosimetry measurements. Obviously, the largest pore size of $1 \mathrm{~mm}$ measured by mercury porosimetry was not observed from micro-tomography as it is larger than the analyzed zone diameter.

The mean porosity and pore sizes detected by this microtomography analysis of a very small analyzed zone of the medium $\left(0.13 \mathrm{~mm}^{2}\right)$ are consistent with values obtained at a larger scale by mercury porosimetry. This may indicate that such a small medium surface area represents rather well the average porous structure of the

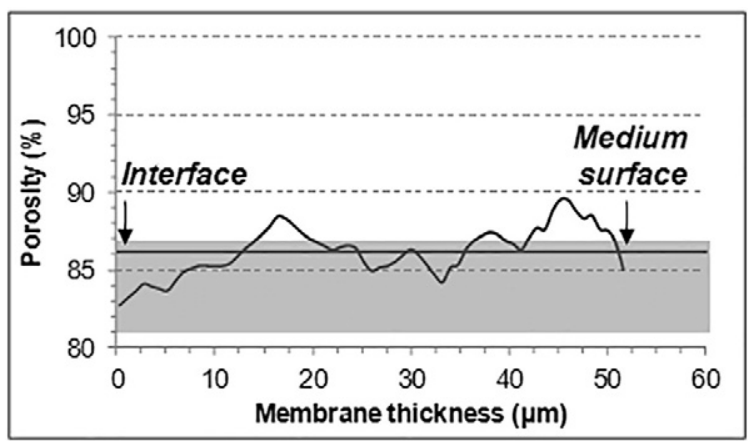

Fig. 11. Through-plane porosity profile of the membrane from micro-tomography analyses carried out on sample CM.

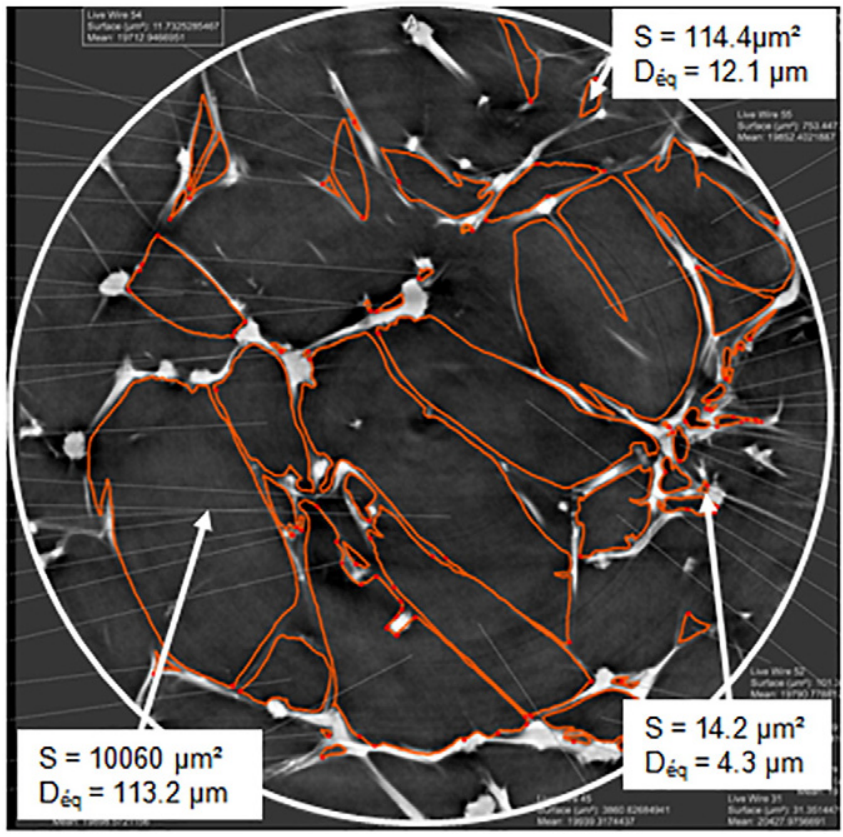

Fig. 12. Example of a $2 \mathrm{D}$ image of the polyethylene membrane used to determine pore sizes.

polyethylene membrane. As for the porosity values derived from micro-tomography analyses of the fibrous support, additional experiments would be required to assess this assumption.

The comparison of results involving different measurement techniques requires that their limitations, and the kind of results that they can determine, are kept in mind. Although mercury porosimetry analyses need a certain pressure level to be applied on samples during mercury penetration inside the pores, which could lead to an underestimation of the porosity, quasi-static pressure steps are assumed, which enable confidence in such measurements. Concerning $\mathrm{X}$-ray micro-tomography, apart from the issue of sample area representativeness, pre-processing steps involved in image processing, and especially the filtering and thresholding steps, could lead to slightly overestimating the void fraction and consequently the porosity. The chosen resolution must thus be consistent with the characteristic size of the solid structure. Finally, it should be mentioned that mercury porosimetry determines hydraulic pore diameters, detected in the three dimensions of space, whereas diameters obtained by the methodology developed to treat micro-tomography data are equivalent diameters, detected only in planes transverse to the flow, i.e. in the medium plane. The good agreement between mean porosity and pore size obtained for the polyethylene membrane shows that, despite all these considerations, mercury porosimetry and X-ray micro-tomography give similar results for this type of medium.

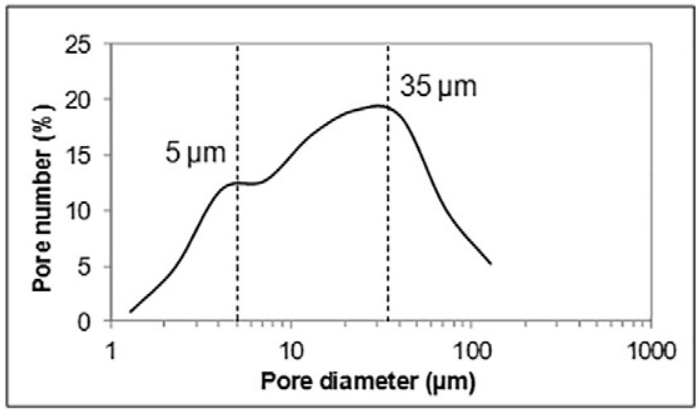

Fig. 13. Pore size distribution of the polyethylene membrane obtained by processing 2D images. 


\section{Conclusion}

The solid and porous structure description of an original two-layered porous medium was carried out using complementary experimental methods, at different scales. The solid structure was investigated by SEM surface observations, X-ray micro-tomography 3D reconstructions and thickness measurements. These methods revealed the distinct solid structures and characteristic lengths of each layer separately. The mean fiber size of the fibrous support was determined by image processing of SEM observations. For this layer, a good agreement was also highlighted between the thickness values obtained by a facility developed for lowpressure measurements on paper samples and by X-ray microtomography 3D reconstructions on similar sample sizes, respectively.

The porous structure was characterized by mercury porosimetry and $\mathrm{X}$-ray micro-tomography to provide complementary information. The three different analyzed surface areas used to carry out these analyses enabled to determine whether mean porosity deviations could be detected. For the fibrous support, different mean porosities were obtained by X-ray micro-tomography depending on the sample surface area. While the porosity obtained on a large sample was similar to that obtained by mercury porosimetry for a similar sample area, a higher value was given by X-ray micro-tomography for an 11 times smaller sample area. This points out the rather in-plane heterogeneous nature of the porous structure of this fibrous support. For the membrane layer, the porosity obtained by X-ray micro-tomography for a small sample surface area was in good agreement with the mean porosity obtained by mercury porosimetry. This indicates that such a small medium surface area rather well represents the average porous structure of the polyethylene membrane. The results obtained by both techniques in terms of pore sizes, and especially the smallest pore size, of the polymer membrane were also in good agreement.

\section{Nomenclature}

$\mathrm{D}_{\mathrm{p}} \quad$ pore diameter, $[\mathrm{m}]$

$\mathrm{P}_{\mathrm{c}} \quad$ pressure, $[\mathrm{Pa}]$

S surface area, $\left[\mathrm{m}^{2}\right]$

Greek letters

$\gamma \quad$ mercury superficial tension, $\left[\mathrm{N} \cdot \mathrm{m}^{-1}\right]$

$\varepsilon \quad$ porosity, [-]

$\theta \quad$ solid/liquid contact angle, [-]

\section{Acknowledgements}

The authors gratefully acknowledge the Lydall company for supplying the Arioso ${ }^{\circledR}$ medium and samples of both its layers separately, which were characterized through this study.

The authors would also like to thank the LGP2 Laboratory (Laboratoire de Génie des Procédés Papetiers, Grenoble, France) for the thickness measurements, the NOVITOM company (Grenoble, France) for the acquisition of micro-tomography data, and the IMN Laboratory (Institut des Matériaux Jean Roussel, Nantes, France) for the SEM analyses.

\section{References}

[1] M.A. Tahir, H. Vadehi Tafreshi, Influence of fiber orientation on the transverse permeability of fibrous media, Phys. Fluids 21 (2009) 101-104.

[2] A.M. Saleh, S.A. Hosseini, H. Vadehi Tafreshi, B. Pourdeyhimi, 3D microscale simulation of dust-loading in thin flat-sheet filters: a comparison with 1-D macroscale simulations, Chem. Eng. Sci. 99 (2013) 284-291.

[3] L. Le Coq, Influence on permeability of the structural parameters of heterogeneous porous media, Environ. Technol. 29 (2008) 141-149.
[4] S.S. Manickam, R. McCutcheon, Characterization of polymeric nonwovens using porosimetry, porometry and X-ray computed tomography, J. Membr. Sci. 407-408 (2012) 108-115.

[5] V. Koivu, M. Decain, C. Geindreau, K. Mattila, J.F. Bloch, M. Kataja, Transport properties of heterogeneous materials. Combining computerised X-ray microtomography and direct numerical simulations, Int. J. Comput. Fluid Dyn. 23 (10) (2009) 713-721.

[6] P. Soltani, M.S. Johari, M. Zarrebini, Effect of 3D fiber orientation on permeability of realistic fibrous porous networks, Powder Technol. 254 (2014) 44-56.

[7] P.C. Gervais, S. Bourrous, F. Dany, L. Bouilloux, L. Ricciardi, Simulations of filter media performances from microtomography-based computational domain. Experimental and analytical comparison, Comput. Fluids 116 (2015) 118-128.

[8] A. Charvet, S. Rolland Du Roscoat, M. Peralba, J.F. Bloch, Y. Gonthier, Contribution of synchrotron X-ray holotomography to the understanding of liquid distribution in a medium during liquid aerosol filtration, Chem. Eng. Sci. 66 (2011) 624-631.

[9] A. Jackiewicz, S. Jakubiak, L. Gradon, Analysis of the behavior of deposits in fibrous filters during non-steady state filtration using X-ray computed tomography, Sep. Purif. Technol. 156 (2015) 12-21.

[10] X. Thibault, J.F. Bloch, Structural analysis by X-ray microtomography of a strained nonwoven papermaker felt, Text. Res. J. 72 (6) (2002) 480-485.

[11] S. Rolland du Roscoat, M. Decain, X. Thibault, C. Geindreau, J.F. Bloch, Estimation of microsctructural properties from synchrotron X-ray microtomography and determination of the REV in paper materials, Acta Mater. 55 (2007) 2841-2850.

[12] S. Ramaswamy, M. Gupta, A. Goel, U. Aaltosalmi, M. Kataja, A. Koponen, B.V Ramarao, The 3D structure of fabric and its relationship to liquid and vapor transport, Colloids Surf. A Physicochem. Eng. Asp. 241 (2004) 323-333.

[13] X. Huang, Q. Wang, W. Zhou, D. Deng, Y. Zhao, D. Wen, J. Li, Morphology and transport properties of fibrous porous media, Powder Technol. 283 (2015) 618-626.

[14] A. Makandar, B. Halalli, Image enhancement techniques using highpass and lowpass filters, Int. J. Comput. Appl. 109 (14) (2015) 12-15.

[15] G.W. Zack, W.E. Rogers, S.A. Latt, Automatic measurement of sister chromatid exchange frequency, J. Hystochem. Cytochem. 25 (7) (1977) 741-753.

[16] A. Zerrouati, M. Rueff, B. Bouchekima, Study of paper transverse shrinkage during thermal drying, Dry. Technol. 331 (10) (2015) 1170-1179. 